
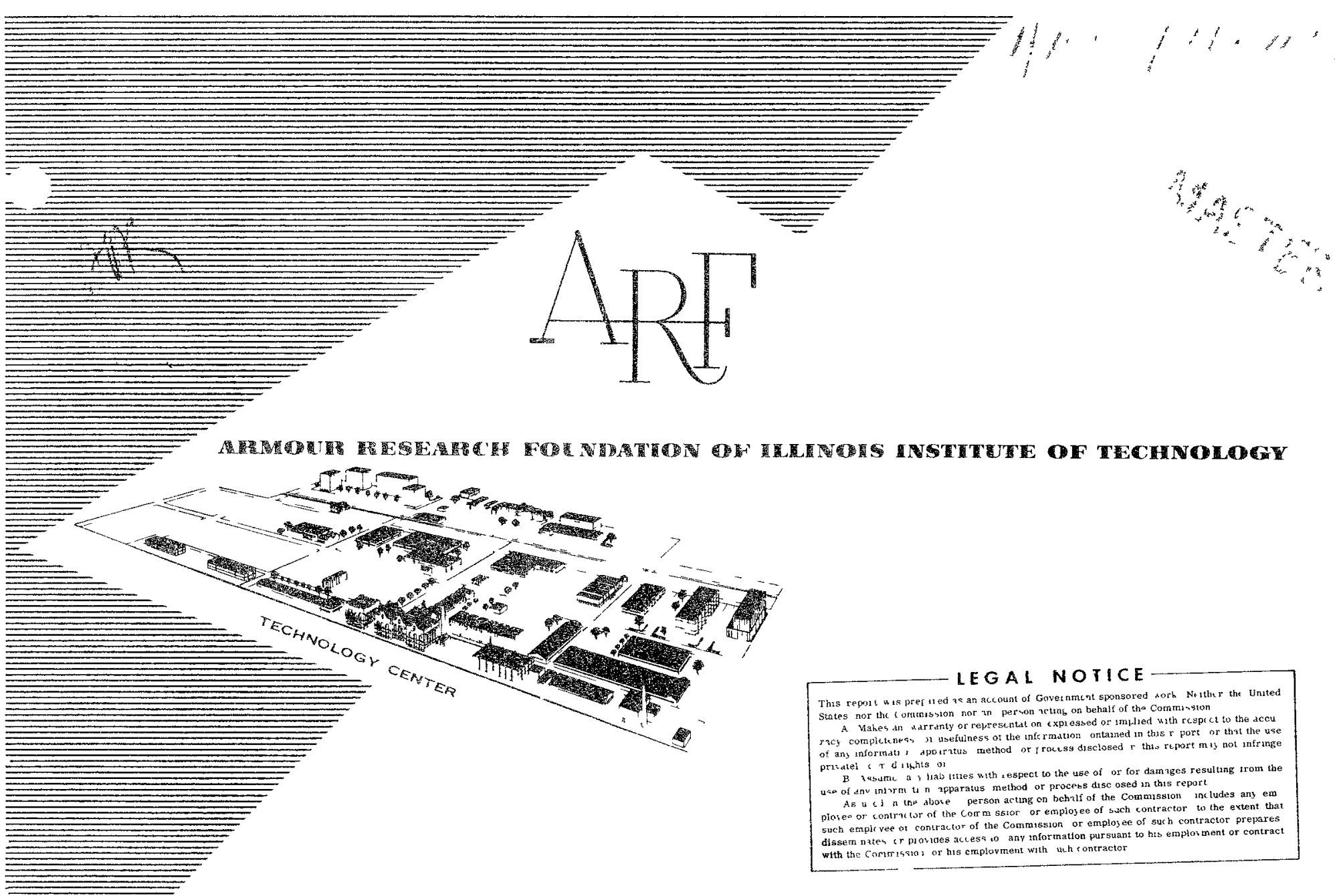

NEW CONCEPTS FOR CONTROL AND USE OF NUCLEAR EXPLOSIONS

Francis B. Porzel

Senior Scientific Advisor

Armour Research Foundation

29 April 1959

Prepared for

American Society of Civil Engineers

Annual Meeting

May 4-8, 1959

Cleveland, Ohio

\title{
MEAEALCHI FOR INIUESTRY
}

ARF Technical Memorandum 421 


\section{DISCLAIMER}

This report was prepared as an account of work sponsored by an agency of the United States Government. Neither the United States Government nor any agency Thereof, nor any of their employees, makes any warranty, express or implied, or assumes any legal liability or responsibility for the accuracy, completeness, or usefulness of any information, apparatus, product, or process disclosed, or represents that its use would not infringe privately owned rights. Reference herein to any specific commercial product, process, or service by trade name, trademark, manufacturer, or otherwise does not necessarily constitute or imply its endorsement, recommendation, or favoring by the United States Government or any agency thereof. The views and opinions of authors expressed herein do not necessarily state or reflect those of the United States Government or any agency thereof. 


\section{DISCLAIMER}

Portions of this document may be illegible in electronic image products. Images are produced from the best available original document. 
This paper describes some new and basic concepts for control and use of nuclear explosions in peaceful uses. These applications involve large scale public works for which the designation "civil engineering" is particu.. larly appropriate.

Because some of the ideas piresented here may seem so novel as to oppose first intuition about them, it probably helps to state at the outset that the field "Fluid Dynamics of Strong Shocks" is literally a new branch of physics. Historically, this field grew out of the military necessity to understand the blast and other hydrodynamic effects of ruclear weapons; it is only fair to add that the progress would not have been possible without the enormous experience invested in that field during the past fifteen years. The military applications clearly could not be paralleled by a similar growth in the nonclassified literature. But the basic concepts axe not classified, they are readily derived from conservation of mass, momentum, and energy and in many respects the most recent developments in this field represent a return to classical mechanics. The field tib characterized by the non-linearity in the hydrodynamic equations of motion and in the equation of state of materials, due to the high pressures and stressos characteristic of such explosions. The end result is an emphasis on different mathematical disciplines and theoretical techniques, new but no mro difficult than their counterparts in "acoustic" theory. Unfortunately, the linearity assumption pervades so many fields of science and engineering - a.erodynamics, elasticity, vibration, electronics - that we develop a corresponding intulition about the behavior of natural processes as if linearity were a universal characteristic of Nature. But in the realm of very large displacements and unbalance of forces the concepts of linearity are more of a liability than an asset. In 
some cases, the linearity assumption literally annihilates the most significant terms in the equations of motion and denies the essence of the problem we are trying to understand. A corresponding confusion would result if we were trying to understand a radio or television set which was built with nonlinear resistances so that electrical resistance was not a constant property of material, independent of voltage and current, but proportional to some high power of the current itself.

This paper is restricted to a discussion of two principle concepts and their applications: waste heat and channeling. 7 The waste heat concept pro- 7 vides a categorical method for the control and use of an explosion by control of the properties of the medium surrounding the bomb or energy source; the applications are possible because of non-linearity of the equation of state for compression. The channeling concept provides a categorical method for control and use of an explosion by control of the geometry of the materialsur-" rounding the energy source; the arplications are possible mostly because of the non-linearity in the shock velocity and in the material velocity behind the shock 3

I. WASTE HEAT

Irreversible heating in a material, caused by the entropy change across a shock which traverses it, is well known. The effect is proportional to the cube of the over-pressure at low pressures and is, therefore, vanishingly s mall in the acoustic approximation. There is a tendency to overlook the corresponding fact then that the entropy change is necessarily a controlling feature of the hydrodynamics at the high pressures which exist close to any rapid energy release. Thus, the fireball from atomic bomb in air is not due to the bomb parts directly but due to the passage of the shock wave over the 
surrounding ajr; if the ajr were adiabatic in compression (let alone its nonlinearity) there would be no fireball from an atomic bomb. Also, the "hole" represented by the bubble of an underwater explosion and the cavity in an underground explosion would exist for other hydrodynamic reasons, but they would be different from their present size.

We define the waste heat $Q(P)$ to be the net energy per unit mass (above initial energy content), which remains in the material (ur 1 , lullly shocked to pressure P) after it has finally expanded adiabatically down to the ambient pressure $P_{0}$ which existed before the explosion. The "waste heat concept" itself has a special meaning with regard to explosions in solids, liquids, and their mixtures with gases; it is a convenient useful and accurate engineering assumption which justifies the subsequent bookkeeping of internal energy by dividing it into "waste heat" and "available energy". The waste heat concept. implies important but deceptively simple differences from entropy change: there would be no waste heat if the material returned to zero pressure instead of ambient pressure. This bookkeeping approximation is justified by the fact that the bulk modulus for compressibility is quite insensitive to temperature and for a variety of other compensating effects both in the process of shock and in the subsequent expansion.

The relative magnitude of several energy quantities and the method of calculating waste heat is indicated in Figure 1. The figure is a conventional pressure-volume diagram; energy is portrayed as an area on such a diagrarn. The full curve is called the "Rankine-Hugoniot curve" which gives the relation between pressure $P$ and specific volume $V$ at various shock strengths. By conservation of energy alone, the total energy added to the material by the shock is given by $E_{T}-E_{0}=P\left(V_{0}-V\right)$. This is the area of the rectangle below the pressure $P$ and between $V_{0}$ and $V$. The kinetic energy $F_{k}$ of the 
the material, with velocity $u$ behind the shock, is given by conservation of mass and momentum alone as $\frac{1}{2} u^{2}=\frac{1}{2}\left(P-P_{0}\right)\left(V_{0}-V\right)$. This is the triangle in the upper right of the total energy rectangle; whatever the actual path during; shock compression, the latter equation shows that the compression is effectively as if the material were shocked along the straight line joining $P_{o}$ with P. It immediately follows that the internal energy in the material is the quantity of $E_{i}-E_{0}=\frac{1}{2}\left(P+P_{0}\right)\left(V_{0}-V\right)$ which is clearly the total trapezoid in the total energy rectangle lying below the straight line joining $P$ and $P_{0}$ Although these energy expressions and the Rankine-Hugoniot relations may be derived in a different manner with more restrictive assumptions, $f$ the simple derivation shown here is sufficient to indicate that these relations are quite general and apply without regard to the equation of state of the material or the details of the shock process.

For solids and liquids the subsequent expansion of the material behind the shock can be described in a simple manner: the material will expand by essentially adiabatic processes back to its original pressure $P_{0}$ and volume $\mathrm{V}_{0}$, and for most practical purposes, will expand along the Rankine-Hugonicit curve. Since the area under that curve is the PdV energy subsequently given up during the expansion, that area is defined as the available internal energy. It follows that the lens-shaped area lying between the straight line and the curve is by definition the waste heat itself. The energy represented by this area appears as a final temperature rise in the material left long after the shock has passed. Heat conduction processes occur on time scales much longer than the rapid process of compression and expansion described here, so the deposition of waste heat is literally forever so far as the shock hydrodynamics is concerned.

For all materials, thexc exists some shock pressure high enough that 
the waste heat is sufficient to vaporize the material irreversibly, even after it has expanded and cooled back to ambient pressure. We define that shock pressure to be the "vaporization" pressure, and the material inside the corresponding radius becomes part of the bomb cavity. The energy represented by the "waste heat area" in excess of the total heat to vaporization appear's as superheat in a gas, is available by expansion, and is not counted as waste heat. Very close to the bomb, well in excess of vaporization pressure, all materials are initially good transmitters.

It ts easy to find or to fabricate materials and mixtures which "crush down" at some relatively low pressure. In that case the RankineHugoniot curve is much more shargly bowed, as shown in the figure at the upper right and nearly all the internal energy appears as waste heat. As a result of the Rankine-Hugoniot relations, all materials compress to some limiting density ratio, like 10 to 1 or 4 to l even at infinite shock pressures. For this reason all materials display a vertical Rarkine-Hugoniot curve at pressures high enough and all materials become ideal absorbers at such high pressures provided they are not greatiy superheated.

At low pressures the curvature in the Rankine-Hugoniot curve is very slight; in the acoustic approximation the Rankine-Hugoniot is a straight line passing through $\left(P_{0}, V_{0}\right)$. This is the domain of ordinary elastic theory. Strictly speaking, the straight line approximation is never really reached, but the waste heat area becomes very small and we speak of material as an ideal transmitter. All materials, at least below their crushing strength are ideal transmitters at low pressures, as show'n at the lower right.

\section{WAVE PROPAGATION}

We discuss briefly the fraction of energy which is hydrodynamically 
available after the shock has run to some appreciable distance $R$ from an instantaneous energy release in a liquid or a solid. The damage and hence the difficulty of controlling an explosion can often be specified in terms of the peak pressure $P$ at the shock front.

At high shock pressures, the usual theory in the current literature is based on certain similarity assumptions, which are not really justified in their own right, but the similarity condition would require that the decay of pressure with distance be as $P \sim R^{-3}$, the quantity $P R^{3} \sim W$, and $\frac{d \ln P}{d \ln R}=-3$. At low pressures the usual description is based on the acoustic approximation and gives $\left(P-P_{0}\right) R \sim W$ and $\frac{d \ln \left(P-P_{0}\right)}{d \ln R}=-1$. In both cases, the total energy $W$ in the wave is taken to be constant and equal to the original energy released. These results, both at high and low pressures, are misleading over-simplifications and the reasons can be summarized briefly by means of the waste heat concept.

With the energy partitioned according to the waste heat concept, the energy balance any time is:

$$
\begin{aligned}
\begin{array}{c}
\text { Effective } \\
\text { Energy }
\end{array}=\begin{array}{c}
\text { Original. } \\
\text { Energy }
\end{array}-\begin{array}{c}
\text { Dissipated } \\
\text { Energy }
\end{array}=\begin{array}{c}
\text { Available Internal plus } \\
\text { Kinetic Energy }
\end{array} \\
W^{\prime}=\dot{W}-4 \pi \int_{0}^{R} \rho_{o} Q^{2} d R=\frac{4}{3} \pi R^{3}\left(P-P_{o}\right) f
\end{aligned}
$$

The validity of this equation $i s$ wholly a matter of definition and conservation of energy. The effective energy is $W^{\prime}$, the energy left to propagate the shock and to do damage at the distance $R$. The extreme right-hand term contains the volume of the shock $\left(4 \pi \frac{R^{3}}{3}\right)$ and the peak pressure $P$ at the shock front, pressure having dimensions of energy per unit volume. The factor $f$ is defined to relate the average energy density per unit volume on the interior of the wave (available internal energy plus kinetic energy) to the peak pressure 
at the shock front. Because the compressional energy and kinetic energy both decrease monotonically on the interior of the wave, the factor $f$ will be less than unity, in terms of the peak energy density at the shock front i,tself; $f$ will be a number like $\frac{1}{2}$ for strong shocks in gases but be a much s maller number when the shock becomes weak. The waste heat concept makes it possible to calculate the dissipated energy by integrating over the past history of the shock front since the functions $P(R)$ and $Q[P(R)]$ are known and the heat is "stored forever" in the material. Because the waste heat $Q$ is always a positive quantity the integral is monotonically increasing. Equation 1 shows that the effective energy $W^{\prime}$ (or its counterpart on the extreme right) is not proportional to a constant $W$ but decreases continually as the shock progresses and the waste heat accumulates in the material left behind.

Assuming that the original energy $W$ is constant (it is not necessary to assume that the energy release was instantaneous) Equation 1 may be differentiated with respect to time or distance $R$ and after suitable manipulations the rigorous result is obtained that

$$
\frac{d \ln \left(P-P_{o}\right)}{d \ln R}=-3\left[\frac{1+\frac{P_{o} Q}{\left(P-P_{o} f\right.}}{1+\frac{d \ln f}{d \ln \left(P-P_{o}\right)}}\right]
$$

At high pressures, $f=$ constant $\cong \frac{1}{2}$ or $\frac{1}{3}, \frac{d \ln f}{d \ln P-P_{0}} \cong 0$ and at the same tirne the quantity $\rho_{0} Q /\left(P-P_{o}\right)$ is its elf close to unity. The result is that the $\log -$ arithmic decay of pressure with distance is two or three times greater than the similarity assumption would indicate and means that the peak pressure decay is like the sixth or perhaps the ninth power with distance from the source. This rapid decay of pressure, say $P \sim R^{-6}$ with distance instead of $P \sim R^{-3}$ is 'w io dissipation of energy through the waste heat process; 
it results in dissipation of energy fractions like 99 per cent of the original W energy before the shock wave reaches the crushing pressure or other pressures of practical interest.

It can be shown at low pressures the factor $f$ is proportional to the s quare of the over-pressure, $f \sim\left(P-P_{0}\right)^{2}$ so that $\frac{d \ln f}{d \ln \left(P-P_{o}\right)}=2$. It follows from Equation 2 that $\frac{d \ln P-P_{0}}{d \ln R}=-\left(1+\frac{P_{o} Q}{\left(P-P_{o}\right)}\right)$. But in the acoustic approximation $Q \sim\left(P-P_{Q}\right)^{3}$ and because $\left(P-P_{0}\right) f \sim\left(P-P_{0}\right)^{3}$ it follows that the correction term $\frac{\rho_{0} Q}{\left(P-P_{o}\right) f}$ (representing the waste heat) does not vanish but approaches a constant limiting value even in the acoustic approximation.

Despite the variations in the factor $f$ with shockstrength and the variation in the behavior of the waste heat with pressure, it is possible to secify the available energy $W^{\prime}$ by a simple relationship with pressure as:

$$
W^{\prime}=W_{1}^{\prime}\left(\frac{P}{P_{1}}\right)^{\frac{1}{2}} \cong \frac{W}{2}\left(\frac{P}{P_{1}}\right)^{\frac{1}{2}}
$$

$P_{1}$ is the "vaporization pressure". The term $W_{1}^{\prime}$ is the available energy at the vaporization pressure and $W^{\prime}$ will usually be about $\frac{1}{2}$ the original energy release $W$. The pressure $P_{1}$ will be in the order of tens of kilobars for most soils but may be of the order of megabars for dense homogeneous materials like concrete or steel if free of voids. In any case, Equation 3 shows why the available energy $W^{\prime}$ will be only a small fraction of the original energy release W, and will decrease with distance.

Some consequences of the waste heat concept on wave propagation follow from such considerations as these.

1. Most of the energy from the explosion, fractions like 99 per cent, is deposited as heat very close to the source, in the cavity and in a thin shell of compressed material immediately surrounding the bomb cavity. 
2. The energy avallabie to produce damage or seismic waves bears no set direct relation with the original energy release; the residual energy fraction depends upon the initial configuration of material and room immediately surrounding the bomb, upon the vaporization pressure and the dynamic crushing strength of the rock or soil. The apparent energy at long distances will be a small fraction of the original energy release, like .001. 3. The concept of energy partition, so much going into the bubble, so much going into blast is essentially a misnomer; the available energy is constantly decreasing fraction with distance and the accumulated waste energy rapidly approaches 100 per cent of the original energy.

4. It is doubtful if the acoustic approximation ever applies, in the sense of a constant energy in the wave. For example, Cole's "Underwater Explosions" gives the universal experimental for TNT explosions in water in the form

$$
\text { the evponent } 1.13 \text { and }
$$

$$
\left(P-P_{o}\right)=\text { constant }\left(\frac{W^{\frac{1}{3}}}{R}\right)^{1.13}
$$

From Equation 2 we recognize that whatever the value of $Q$ and $f$ at low pressures, the limiting value is such that $\rho_{0} Q /\left(P-P_{0}\right) f=0.13$.

5. If the attempt is made to measure the energy release from an explosion by measuring the kinetic and compressional energy in the shock at appreciable distances from the source, the results are bound to indicate very s mall energy releases perhaps in errop by a factor like 100 from the original energy release. Such procedures have been used by a number of agencies, say in attempting to evaluate the energy release from metal-water reactions suspected to occur in reactor explosions; the results indicated very small energy releases. The point is that a similar measurement from a TNT 
explosion would also lead to similarly small values of energy, entirely inconsistent with the known energy release of TNT, as used for scaling. Damage is usually predicted on the basis of $\mathrm{W}^{1 / 3}$ scaling from TNT results, and this "apparent energy" grossly underestimates the damage.

\section{UNDERGROUND EXPLOSIONS}

A typical history from an explosion in soil displays striking features, indicated in Figure 2, which would not be suspected on the basis of either linearized or conventional shock theory. This description is the author's interpretation for what should have happened and what did happen in the Rainier underground shot at Nevada Test Site in September 1957; the reader is reminded that the interpretation is not yet universally accepted.

The plot of shock velocity versus distance as in Figure 2 is convenient to describe the various phases in the history of the shock growth. At high enough pressures, conservation of mass and momentum alone requires the shock velocity to behave as $U \sim \mathrm{P}^{1 / 2}$ for any medium. The similarity condition $P \sim R^{-3}$ would require $d \ln U / d \ln P=-3 / 2$, which is a straight line of slope $-3 / 2$ on these logarithmic coordinates. At low pressures the shock velocity assymptotically approaches the constant, sound velocity; the general behavior according to conventional theory is shown as the dashed line in Figure 2b. For the purpose of exposition now, we assume the RankineHugoniot curve has the shape shown in the pressure-volume diagram of Figure 2a. The corresponding regions of growth in Figures $2 \mathrm{a}$ and $2 \mathrm{~b}$ are shown by labelling portions of the curve A, B, C, D, and E. The region A is shocked well above the vaporization pressure of the material. The m aterial behaves like a gas because the material behind it is irreversibly vaporized and superheated. But the shock velocity curve decays more rapidly 
with distance, than $U \sim R^{-3 / 2}$ (11ke $U \sim \mathrm{P}^{\mathrm{l} / 2}$ ) because of the enormous energy expended on latent heat of vaporization of the material. The region $B$ corresponds to the range of pressures between the pressure at which 100 per cent of the material is irreversibly vaporized and some lower pressure at which all of the material can be heated to the boiling point by waste heat but too weak to vaporize any of it. $B$ is a region of moxe or less constant temperatures. For other reasons also, the peak pressure is probably constant, and in any case, the shock velocity runs constant for some distance. In Rainier, this constant velocity region extended for all material which originally lay between 5 meters out to 17 or 18 meters from the bomb. The regions $C$ and $n$ shown are best understood by noting that the RankineHugoniot equation for shock velocity requires :

$$
u^{2}=v_{0}^{2} \frac{\left(P-P_{0}\right)}{V_{0}-V}
$$

This equation means that the straight line joining $P$ and $P_{0}$, as shown in Figure 1, represents the square of the shock velocity, $U^{2}$. Ambient sound velocity has been indicated by the dashed line on Figure 2a, as an extention of a straight line portion of the Rankine-Hugoniot curve at low pressures in the region $\mathrm{E}$. The region $\mathrm{C}$ corresponds to those pressures below vaporization but at which the shock is still sufficiently strong to be supersonic with $r$ espect to ambient sound velocity. The region $D$ is particularly interesting because it represents a region of pressures well above the crushing pressure but in which the shock velocity is below ambient sound velocity. In this region of pressures, precursor sound signal may precede the main shock pulse, but they are necessarily insignificant in strength compared with the main shock pressure. The region E represents the range of pressures below the crushing strength of the rock. By virtue of the sumplification used to portray 
compressibility in the region $\mathrm{E}$ on Figure $2 \mathrm{~b}$ and in the corresponding region $\mathbf{E}$ on Figure $2 a$, the crushing pressure is a horizontal straight line.

We note that acoustic theory would require that the entire history of the explosion would be represented by straight lines, both for the compressibility in Figure $2 a$ (dashed line) and a constant value (sound velocity) represented by $\mathrm{E}$ in Figure $2 \mathrm{~b}$.

Some comments on the history of an under ground explosion are as follows.

1. There is some meaning to a "constant energy" in the acoustic approximation in the region $E$, which is by definition beyond the crushed zone, a distance like 100 feet for one kiloton in a medium like Nevada soil. Surprisingly enough, Nevada rock is a relatively good transmitter of shock energy because of its high water content at depth. Energy fractions like one per cent of the original energy may still be available in the seismic wave at this distance, which would be significant if the material beyond the crushed zone were completely homogeneous and elastic.

2. The existence of faults, joints, fissures, and free-surfaces all constitute mechanisms whereby the compression (due to the shock transmitted beyond the crushed zone) is readily relieved (at the expense of the net energy transmitted further out in a seismic wave). As such, the "earth quakes" generated by these explosions are more or less coincidental to the shock process and their relationship to the energy release is ambigious. Both the Rainier event (1957) and the Hardtack II events (1958) resulted in displacements like two feet along joints or planes of structural weakness originally in the rock, much like the slippage along an earthquake fault. Very large amounts of energy may be represented as potential energy from a fault block lying above some plane of structural weakness, and the passage 
of the shock may literally trigger this energy. In any case, the subsequent movement of the block (which may relieve the shock stress by moving the block as a whole) generates a disturbance along the induced fault entirely comparable to the mechanism in an ordinary earthquake, but of a much smaller magnitude of energy. It is suggested that the seismic signals alleged to be observed as a result of the Rainier event - if they had any significance at all were probably due to these minor induced earthquakes. It is of considerable reassurance, therefore, in worrying about earthquakes from underground explosions, to note that earthquakes did occur but were very minor events. It is entirely probable that once a test site has been "fired in", by relieving the eons-old accumulation of stresses along planes structural weakness, no further serious disturbances would result from subsequent shots, even like these very minor events.

3. Finally, it is noted that the typical disturbance produced by the shock wave itself is of short duration and of an entirely different nature from the train of waves associated with slippage along a fault block, of relatively very long time duration. There is no strong reason to suppose that the initial compression wave in rock is different from any other shock: a sharp rise followed by a more or less rapid and assympdtotic decay back to ambient. pressure. In the low pressure approximations, the time decay of pressure just behind the front in such a wave can be derived explicitly in. terms of the peak pressure, the time $t$ from the source, and the properties of the material according to

$$
\frac{\partial P}{\partial t}=\frac{P_{0} c_{o}^{2}}{t}
$$

Here $\rho_{o}$ is the density and $c_{o}$ is the sound velocity. Even gross considerations with this equation will. show that the "positive duration" for pressure in the 
shock wave is likely to be of the order of milliseconds; this duration is probably too short for the average seismic instrument to resolve; what the instrument saw was the mountain settling slowly after its initial jolt.

\section{BLAST SHIELDS}

The blast shield is a device intended for very general use as an attenuator of shock energy. In containment of nuclear reactors against internal explosion, the shield is used to protect large critical structural members from excessive shock pressures and loading. The shield usually consists of a series of layers, "ideal absorbers" separated by dense plates. The shield effects a bona fide conversion of hydrodynamic energy into thermal energy through the waste heat principle. Many materials are suitable as absorbers; some of which have been used are redwood, celotex, aerated concrete, and vermiculite concrete.

Figure 3 illustrates some features of pressure decay through several absorbers. Close to the source the medium surrounding the core of the $r$ eactor may itself be an adequate absorber; water is an excellent absorber down to pressures like 20,000 atmospheres. At some pressure levels too low to provide a further effective absorption by the reactor material, or wherever the geometry of the reactor requires, a weaker and more absorbing layer like "A" is introduced. The thickness of the layer is limited by considerations of the partition between internal and kinetic energy, the energy in the explosion and the pressure level at which absorber A reaches its crushing strength and hence cerses to be efficient for absorption. Steel plates are interposed between layers partily to prevent breakthrough of energy by channeling processes, to reflect part of the energy, to flatten out the shock front and to make the shock act more like a smooth piston on a subsequent 
layer. Absorber $B$ is a still weaker matersal than $A$, designed to be suitable for absorption at the pressure level expected after the shock passes through absorber A.

A large scale program supported by the Atomic Energy Commission is in progress at Armour Research Foundation to investigate the optimum materials and design of blast shields. This work includes a detailed study of the space and time history of the material in the absorber under shock loading. The effectiveness of shielding materials is shown in experimental results whereby in some cases the shock completely dies out in passing through the absorbing layer.

Strictly speaking an optimum shield can be tailor made to any given pressure level and energy content. The figure is intended to show that whatever shield is constructed (in which the energy release does not vaporize some of the absorber) it will be more efficient at higher energies and pressures than at low energies. It will be less efficjent for smaller explosions but the shield will always reduce the pressures to some lower, safer level than from a larger explosion. As shown in the figure the blast shield approaches a pressure gate for the pressure level at the output of the blast shield.

An early blast shyeld consisting of redwood and steel laminations was designed by the author in 1955 for the prototype Boiling Experimental Reactor BER-I, of Argonne National Laboratory presently in operation at Lemont, Illinois. At the recent Geneva Conference, "Design Consideration in Nuclear Merchant Ships" UNP 1023 by Goodwin and Worf, shows another application of the redwood and steel blast shield in the US nuclear powered merchant ship Savannah, in which the primary function of the blast shield is a "collision mat". 
V. CONTAINED UNDERGROUND NUCLEAR EXPLOSIONS

An early paper, "Some Peace Time Uses for Nuclear Weapons" (ARF Technical Memorandum No. D-1 by the present author, September 1957) des.cribed an underground power station in which a nuclear weapon could be used as a steam generating plant.

The feasibility of such a scheme rests on the surprising degree to which nearly all the energy of a bomb is converted into heat by the waste heat process within a relatively thin shell - like a few meters - of compressed soil surrounding a bomb cavity. The cavity radius is relatively small, like 15 meters for a small atomic bomb. The essential idea here is to tap that heat energy by introducing water into the cavity and removing it as steam. It should be recognized the outset that the attempt to employ the pressure from an atomic or thermonuclear explosion directly - as has been suggested elsewhere - is inherently infeasible: the energy in the form of hydrodynamic pressure is simple too difficult to contain within a reasonable volume. The basic reason for the feasibility of schemes using the waste heat concept is implied in the mechanical equivalent of heat itself, and in the low energy required to produce high pressures in solids and liquids. As an example, the same energy density which produces a thisusand atmosphere shock in concrete is enough to raise the temperature a sma 11 fraction of a centigrade degree. Conversely an enormous reduction in hyd:-odynamic pressure can be achieved if that energy can be converted into a temperature rise.

Two difficulties arise with this scheme of an underground station, but neither appear to be insurmountable. The first difficulty is the collapse of the arch above the cavity. The early paper suggested the use of a cylindrical cavity instead of a spherical one, in which the cylindrical cavity was to be produced by the bomb itself using the channeling concept to be described 
later. In a recent article by Dr. Teller in "This Week" magazine, a national newspaper supplement, the illustrations showed a similar model of the underground power-plant in which the collapse of the cavity was circumvented by inclining the steam outlet pipe from the vertical. A second difficulty with underground cavities arises because the water content of most soils result in "decrepitation" of the soil similar to the process of making puffed cereals. The vaporization temperature of the soil is, therefore, essentially the boiling point of water and the steam produced would be of too low a grade. One would require a very dry soif of high boiling point to produce high quality steam.

A principle merit in these considerations of underground explosions is because the waste heat concept appears to be the only presently known method whereby thermonuclear power can be made available. Although the familiar fusion machines are eminently superior as a device for general use, it may take until the end of our present century - as Dr. Teller suggested at Geneva - before they become practical. The underground explosion involves no such "scientific breakthroughs" and appears to be largely a matter of engineering development. In any case, the device would be for some special situation where the large amounts of power were required on a temporary basis in a remote location.

\section{CAPSULES OF CONTAINED ENERGY}

A United Nations paper, UN 2178, "A New Approach to Heat and Power Generation from Contained Nuclear Explosions", first presented at the American Power Conference in 1958, suggested a method by which one might obviate the cavity problem entirely, perhaps the necessity for an underground station, by confining the explosion within a spherical steel containing shell of 
reasonable thickness and diameter.

The capsule employs two developments of the waste heat concept: one involves superheat and the other involves a method of mixtures in the medium. From an earlier consideration of the "Thermodynamic Properties of Two-Phase Mixtureg", an informal study, it was found that enormously effective "ideal absorbers" could be fabricated by mixtures lying in the region between what we presently call gases and solids. For all practical purposes, such a mixture is a highly polytropic gas in which the energy required to produce a given pressure is many factors of 10 above that required for ordinary gases, and ordinary gases are factors of thousands more effective than solids or liquids for the storage of energy by compression. So much can be accomplished by the use of mixtures as ideal absorbers that it appeared reasonable to consider "tailormaking" the medium surrounding the bomb and containing it within steel shell. The second development of the waste heat concept is based on the observation that close to the bomb the latent heat of vaporization is a bona fide energy sink and only the excess energy (above this waste heat) appears as superbeat to drive the shock to further distances. Near an atomic bomb the energy density is high enough to vaporize anything but one would like to find a material so dense and so incompressible, with so high a vaporization temperature and so high a heat of vaporization, that the initial shock wave pxoceeding outward from the bomb was just sufficient to raise the material to the vaporization point and no more. These two considerations, mixtures and superheat, dictate the design for layers outside the inner shell: to adjust the medium so that the shock pressure at a given distance is always just at the local vaporization pressure. With such a design, pressure decay, like $\mathrm{P} \sim \mathrm{R}^{-7}$ or $\mathrm{P} \sim \mathrm{R}^{-9}$ as suggested earlier, could be maintained almost indefinitely to large distances and low pressures. 
The paper UN2178 investigated this possibility using graphite, although ordinary silica offers the same kinds of advantages. The total heat of vaporization of graphite is 13,000 calories per gram, about 27,000 calories per $\mathrm{cm}^{3}$. A kiloton explosion would stop vaporizing graphite about five feet out from the bomb, and at this point half the energy would already be lost by conversion into heat in the energy sink represented by the latent heat of vaporization. For one kiloton, the calculations in the paper indicated a steel shell about three inches thick, about twenty feet in radius, at which distances the transient shock pressure would be reduced to about 150 atmospheres. Only the inner few meters in radius would be vaporized carbon, after the detonation the outside layers would be melted or incandescent compacted carbon. But the Clausius - Clapeyron equation itself shows that vaporized carbon is not a frightening material to contain: By definition, graphite at the normal boiling point is confined by standard atmospheric pressure. Figure 4 shows several capsule models: one by adjusting the mixtures as was described, another to accomplish the same purpose by using different materi als and a third which suggested a method for breaking up the bubble on the interior of the cavity.

It appears possible then to contain a kiloton of energy in a remarkably simple furnace: substantially a rigid block of commonplace materials, incandescent throughout most of the interior but with a cooler and specified temperature at the surface. Such a sphere, equivalent in energy to hundreds of tons of coal, would make nearly 100 per cent of the rated energy release thermally available for conversion to heat and power, be at small pressures or confined in a vessel at a few atmospheres, and present no - or trivial problems of radiological hazards, flue gases or disposal of waste. The capsules idea suggests, with the common sense of performing sufficient 
prior tests - that it probably would be possible to detonate a kiloton atomic bomb above the ground in an open city within the volume the size of a small building with no possibility of detecting the explosion - by hydrodynamics, by fall-out or through prompt radiation - beyond the confines of the building in which the capsule was housed.

Combinations of the capsule idea and the underground explosion idea $r$ eadily suggest themselves. Ordinary water has a high heat capacity and even striking experimental evidence exists that ordinary snow fulfills the properties of an ideal absorber very nicely. Based on results obtained by my friend, Dr. Thomas C. Poulter, of Stanford Research Institute, Scientific Director for the Byrd Antartic Expedition in the early 1930's, one estimates that a megaton explosion in snow might be confined at depths like 2,000 feet and that the shock strength might be reduced even below audible levels at distances like five miles. The implications regarding "detectability of nuclear tests" are quite clear.

\section{CHANNELING}

By channeling we mean that the tendency for shock energy to propagate preferentially into rarified media instead of dense media is so pronounced that the energy can be directed almost at will by means of open channels:

The basic hydrodynamic reasons for channeling can be simply shown. Compare the rate of work $\dot{W}$ per unit area and time done by a shock front on the gas compared with that done on a solid medium, assuming the configuration shown in Figure 5. The rate of work $W$ in any medium is given by the product $\dot{W}=P u$, a piston moving with the force $P$ at the material velocity $u$. If the peak pressure at the shock front at a gaven time is the same in the two media - and the hydrodynamics will nearly always guarantee that it is - then 
the rate of work in either medium is described by the equation:

$$
\dot{W}=P u=P\left(P-P_{0}\right)\left(V_{0}-V\right)
$$

Under the condition that the shock pressure is equal across the interface, the ratio of the rate of work in each media is readily obtained as

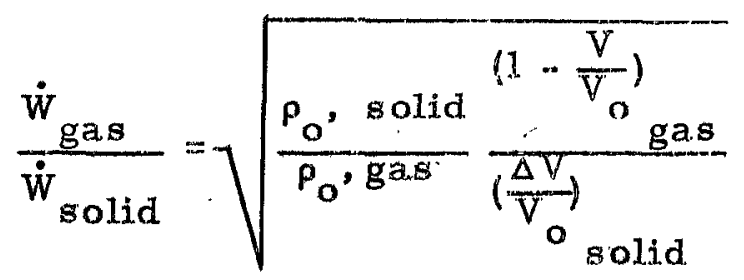

A factor of $10^{3}$ to $10^{4}$ or so occurs in the density ratio between the two media and is alone sufficient to introduce a factor of 30 to 100 for energy propagation in favor of the gaseous phase, even at high pressures where the compression in the solid is comparable to that in the gas. However, over a large range of pressures, $\frac{V}{V_{0}}$ in the gas is so substantially less than 1 so that the corresponding term in the numerator is practically 1. At the same time the density change $\frac{\Delta V}{V_{0}}$ in the solid is very small and this small fractional change in specific volume in the solid again introduces many factors of 10 to the relative rate of work in favor of the gaseous phase. At low enough pressures the relative rate of work in Equatiory 8 approaches the ratio of the familiar "acoustic impedance"

$$
\frac{w_{\text {gas }}}{W_{\text {solid }}}=\frac{\left(\rho_{0} c_{o}\right)_{\text {solid }}}{\left(\rho_{0} c_{o}\right)_{\text {gas }}}
$$

which is the ratio like 10,000 times.

Such considerations show the exormous tendency for the shock energy to literally spill out into the gaseous phase .. a factor of 1,000 at most pressure levels - with a corresponding depletion of the shock energy in the solid or liquid phases. 
VIII. CONTROLLED EXCAVATIONS

Despite the well-known condition that the sound velocity in solid and liquid media may be five to ten times the sound velocity in air, a reverse situation occurs at nearly all pressures great enough to be a practical. interest for excavations.

A strong tendency for the shock in the gaseous media to outrun the shock in the dense media is indicated by considering the equation for shock velocity given in Equation 5. Assuming we have equality in pressure across the interface as in Figure 5, the ratio of the shock velocity in each media would be given by

$$
\frac{U_{\text {gas }}}{U_{\text {solid }}}=\sqrt{\frac{\rho_{0}, \text { solid. }}{\rho_{0} \text { gas }} \frac{\left(\frac{\Delta V}{V}\right)}{\left(1+\frac{V}{V_{0}}\right)}}
$$

When the shock strength is high enough to achieve appreciable compression of the solid (which is widely significant if the solid contains any appreciable air fraction) then the shock velocity in wir outrurs the shock velocity in soil by the square root of the density ratio. A factor of about 30 to 1.applies for many pressures of interest. At low enough pressures the shock velocity ratio must obviously approach the ratio of sound velocities in each media, a ratio in favor of increased shock velocity in the solid medium. However, over the large range of pressures in which the compression in solid is s.mall enough to be considered linear. lalthough much too strong for the linear approximation to apply in air) the ratio of shock velocities behaves as

$$
U_{\text {air }}=\frac{C_{0} \text { air }}{U_{0} \text { solid }} \sqrt{\frac{6 \frac{P}{P_{0}}+1}{7}}
$$


From this equation it is clear that a shock in air will outrun the shock in solids at pressures above 30 atmospheres or roughly 500 psj. Close in, , the ratio reaches the limit like 30 to 1 suggested by the square root of density ratios alone in Equation 10. This result means that the angle of inclination of the ground shock in Figure, 5 is very flat; the shock in ground moves $1 / 30$ the distance outward from the channel as the front moves forward in the channel. For practical purposes, the result is nearly cylindrical symmetry. As a result of such comparisons of shock velocity and with considerations of the detailed material velocity in each of the solid and gaseous phases, it is possible to derive some general criteria for the conditions under which such a channel may be stemmed or plugged. The details are more complex than can be shown here but the results are shown qualitatively in Figure 6. Figure 6 a represents the before and after pictures for an open channel. Categorically, any open channel in a radial direction to the bomb will always open up. The criteria for stemming depend upon the physical properties of a plug shown in Figure 6b. But a general rule of thumb appears that a plug with the same length as the diameter of the channel will usually be sufficient to stem the channel as shown. The hydrodynamic $r$ eas ons are as follows.

When the shock first emerges from the far side of the plug, an enormous rarefaction in shock strength occurs, reducing the transmitted s hock in the channel by a factor roughly equal to the density ratio between the gas and the solid phase. A corresponding rarefaction moves backwards through the plug. The pressures in the solid phase are not similarly reduced and the walls of the channel begin to move in toward the center, closing the channel. If the plug is too thin, the subsequent rarefaction back to the bomb chamber occurs with sufficient rapidity that the bomb energy breaks through. But if the plug is long enough the channel may already be closed 
before the breakthrough can occur. The net result is a continual closure of the channel. At the same time it will be clear from the figure that a pinch jet will almost always move down the channel, ahead of the ground shock because of the continual closure of the air channel behind it. The process is somewhat analogous to the boyhood trick of shooting watermelon seeds by squeezing them.

Surprisingly enough, despite the very much lower efficiency of a nuclear explosion with spherical symmetry in a homogeneous soil, the channeling concept provides the means to make nuclear explosions more efficient than TNT for many excavation purposes. The argument is as follows. Because of the close similarity of density between TNT and most rock-like materials theinitial shock transmitted outward from a tamped TNT charge is of the same order as the internal pressure initially in the high explosive, about 300,000 atmospheres. This results in the shatterin. by TNT with a large loss in energy due to crushing and waste heat within the rock, although of course, not comparable to the waste heat of a tamped nuclear explosion. It is difficult to exploit channeling with TNT even with an open channel because the large mass effect of the TNT is itself almost equivalent to the stemming plug described above. No such mass effects exists for nuclear explosions; by the channeling process it appears possible to channel the energy into very large volumes of air, perhaps reducing the pressures down to levels like 500 psi quoted above, at least down to the crushing strength of the rock, or its yield strength in situ. Thus, because the pressure can be degraded quickly, the energy from a nuclear explosion can be made to lift the overburden with greater efficiency than is possible with TNT.

The area of a cylindrical shock front in soil $(2 \pi r \ell)$ relative to the area 
of the channel itself $\left(\pi r^{2}\right)$, together with the ratio $\frac{l}{r}=30$, is sufficient to indicate that the area of the ground shock is about 60 times the area of the shock at the front of the jet. This means that despite the larger rate of work per unit area in air a large fraction of the energy from an explosion can be directed through the jet to work the ground: the explosion behaves kinetically like a line charge.

The argument has often been advanced that an open radial channel from an explosion will close itself because of the greater sound velocity in dense media than in air. That this argument is invalid $y^{\prime}$ at pressures of interest is clear from Equations 10 and 11. Furthermore, in a porous rock or soil, the sound velocities may actually be comparable. In any rase, Figure 2 a shows that the shock velocity in a crushable dense medium can be quite low even where the pressure exceeds the crushing strength. Hence, the argument for automatic closure applies only at pressure levels which are too low for the channel to crush down. The argument for closure is tanta mount to the statement that a pipe leading from a suddenly pressurized boiler will implode because of the high sound velocity in steel.

\section{REACTOR CONTAINMENT}

For a more complete discussion of reactor containment techniques, the reader is referred to UN 434, "Some Hydrodynamic Problems of Reactor Contaiment", and to various analyses of specific reactors done by Armour Research Foundation listed therein. The most comprehensive of these reports was "Design Evaluation of Boiling Experimental Reactor in Regard to Internal Explosion", (1956) by the author, which was subsequently reproduced as ANL 5651 by the Argonne National Laboratory (1957).

Figure 7 is taken fron UN 434, and illustrates the features of interest 
for containment for one type of reactor, the pressurized boiling water reactor. The explosion is supposed to occur in the core. Without considering the detailed history of the explosion growth, the figure illustrates the major concepts of the present paper. The waste heat concept is illustrated by the blast shield shown, which protects the vertical beams of the hold-down assembly from the initially high shock pressures; these beams would otherwise fail through bending moments. Channeling provides the eventual relief of pressure in this vessel, which relief was designed to occur by an early failure of the concrete below the pressure vessel. The energy was dumped into the air space below the reactor, thenge along the sides of the vessel into the large reservoir of air above the reactor floor, and finally contained within the essentially hemispherical outer containment shell.

X. A SUMMARY OF APPLICATIONS

Aside from the very wide application of these concepts such as in reactor containment, a number of applications for the concepts are summarized below. Some are unique; no doubt, many others could be suggested. In some cases below, the present figsion weapons or the present fusion weapons could be used. In other cases, the suggested use predicates the existence of a small yield, clean, thermonuclear weapon. In some cases, the use predicates the recovery of a by-product from the core of the capsule.

1. Power source for driving a heat engine.

2. Heat source for driving a heating plant.

3. High temperature batch smelter.

4. Cheap production of isotopes.

5. Power source on planets or remote places using natural soil as the "absorber fuel". 
6. Provide new chemical fuels and industrial materials, not possible to produce with present refractories or techniques.

7. Safe and convenient nuclear tests.

8. Uses up stock-pile of fissionable material on peace time applications.

Because the hydrodynamic techniques for containment and controlled excavations are similar and cross -feed each other, one should restate these possibilities for completeness.

9. Harbors, canals, roadbeds, tunnels, mines, oil fields, and large road cuts.

10. Climate control, by canals to make inland seas, or to divert ocean currents through existing land blocks.

11. Control of natural earthquakes, by relieving seismic stresses before they become dangerous.

12. Diversion of lava flow from volcanoes.

In concluding this paper on control and use of nuclear explosions, one restates that the purpose was to invite attention to the existing possi- , bilities, not to sell capsules, underground power stations or a new confederation of nuclear blasting contractors. There are undoubtedly many far better available sources in the world for heat, power and energy than from contained nuclear explosions. Nuclear explosions do possess unique capabilities for pressures and temperatures which ordinary chemical fuels will never make available, and they are a cheap source of energy when one can use them. In reviewing the list of applications, one notes that they are mostly "special applications", for which the unique capability cannot be denied. But since it has become technically true also that thermonuclear war risks race-sulcide, it will be very difficult - perhaps impossible - to keep politics 
and science in separate compartments where such large federal budgets and such large national emotions are so inherently interwoven. Nonetheless, it would be most unfortunate if these applications either were oversold or undersold for reasons which were more political than scientific, Not the least of the new concepts brought into the world by the advent of nuclear weapons will be to liquidate the time-honored separation of science from politics. This forced marriage demands civic responsibilities on the part of scientists for what they create, whether they like to accept those responsibilities or not. It is in many respects a different world from the one we were born in. There is no turning back on nuclear energy, nor is there turning of the back on them; it is here to stay, for good or for evil. The author hopes he has not been misleading by undue optimism, but he also hopes that these concepts will spark the reader's imagination with the fascinating array of forls which wid] be at our disposal once we learn row to live with nucledr energy in the world. 

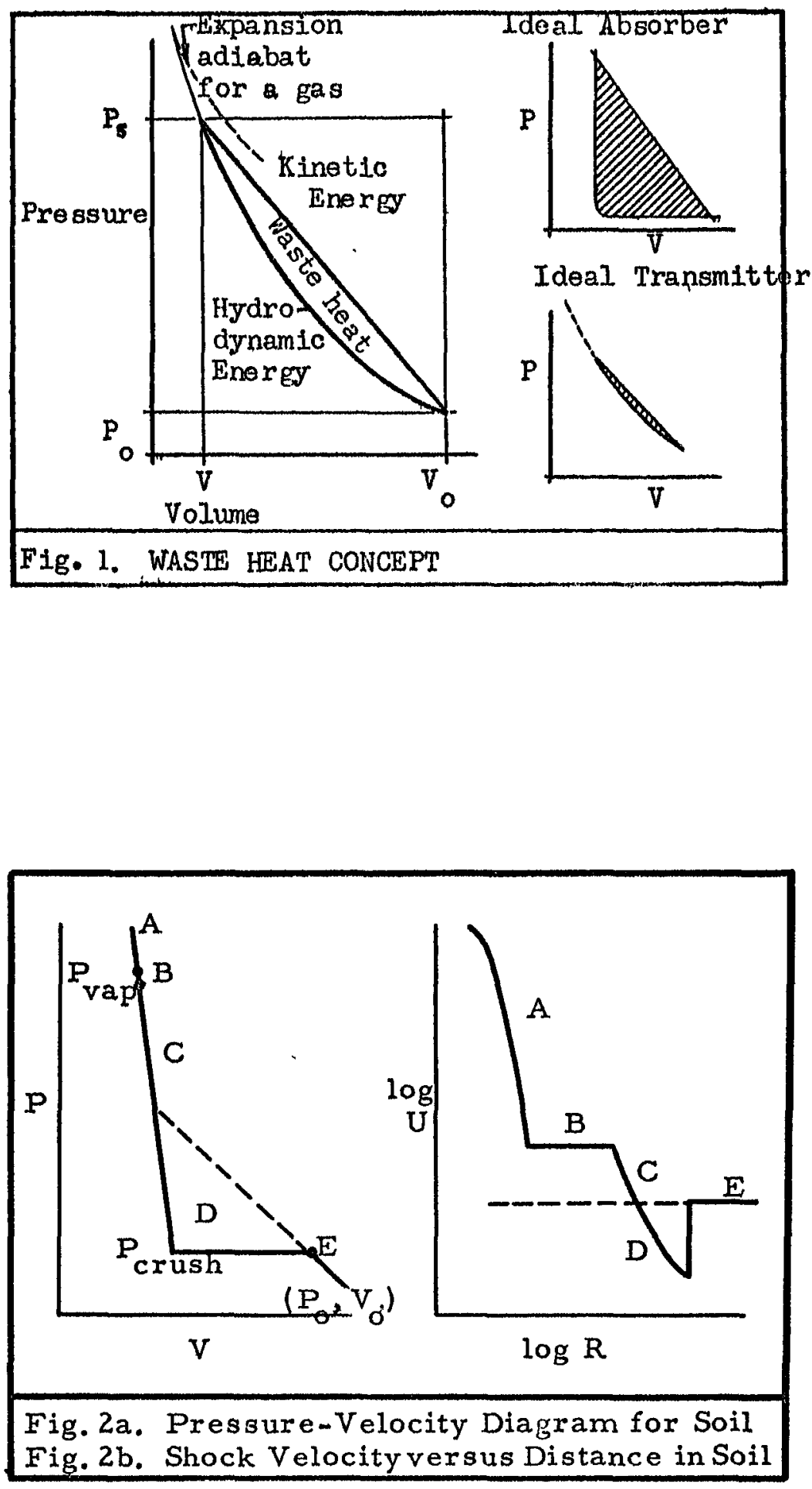

$-29=$ 


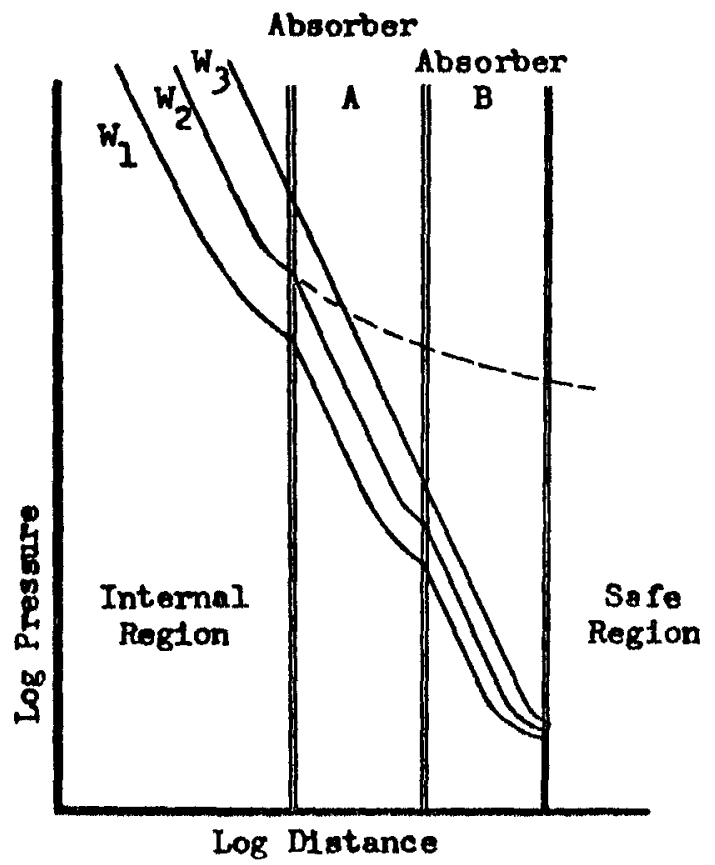

F1g. 3. BLAST SHIFLD CONCEPT

Vortical Scale Compressed for Convenience in Presentation.

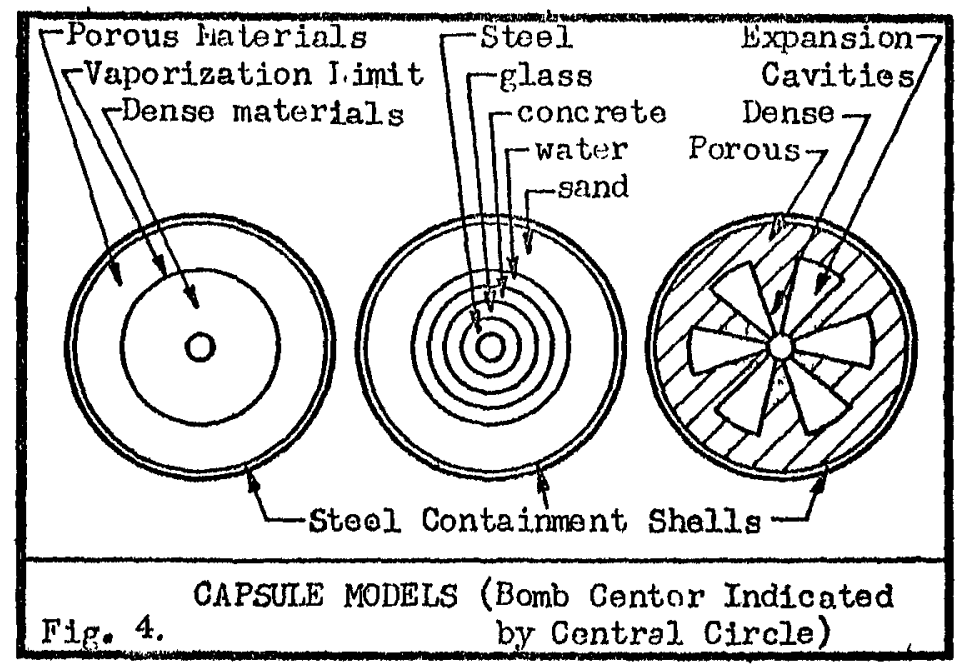



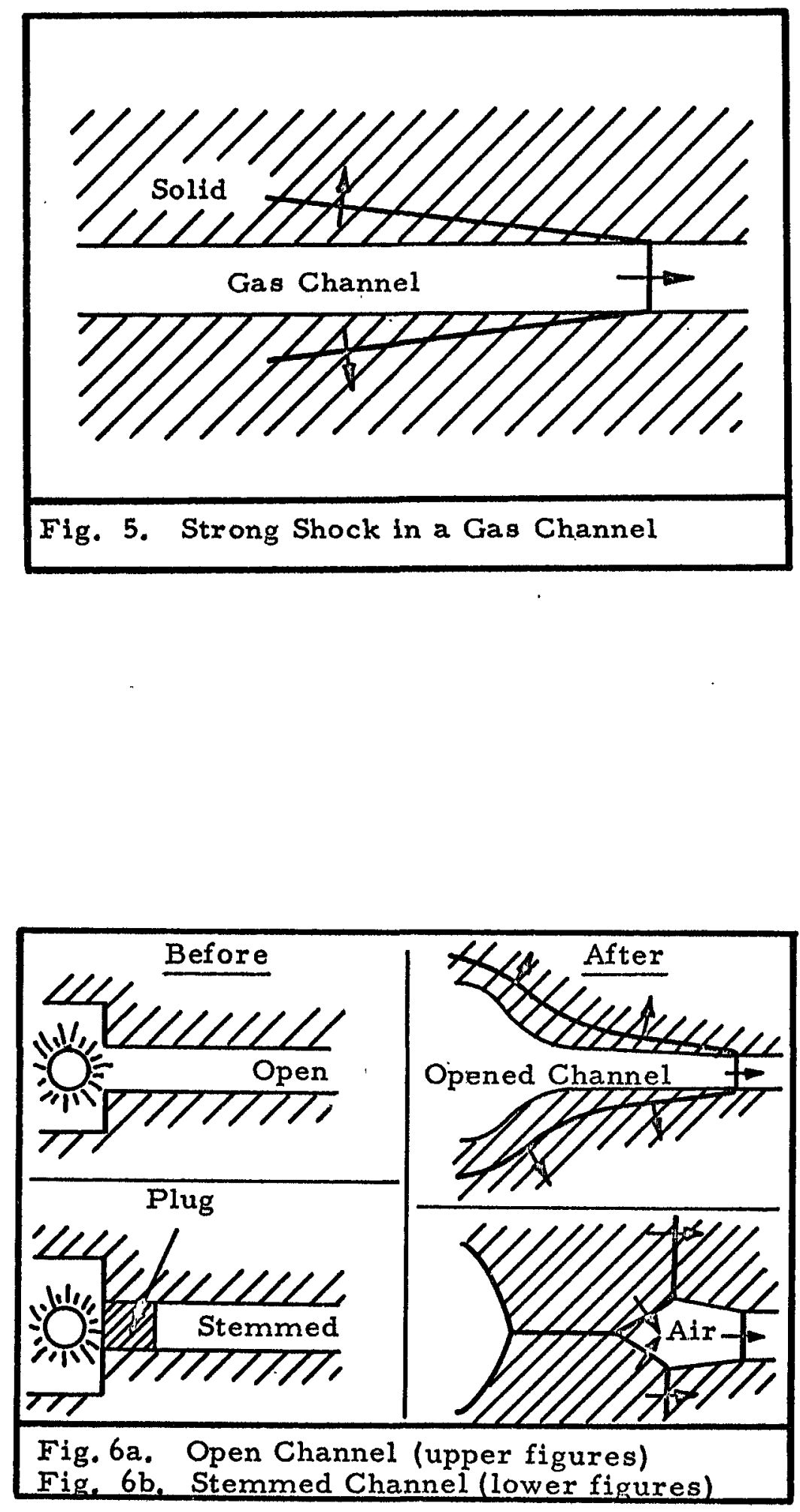


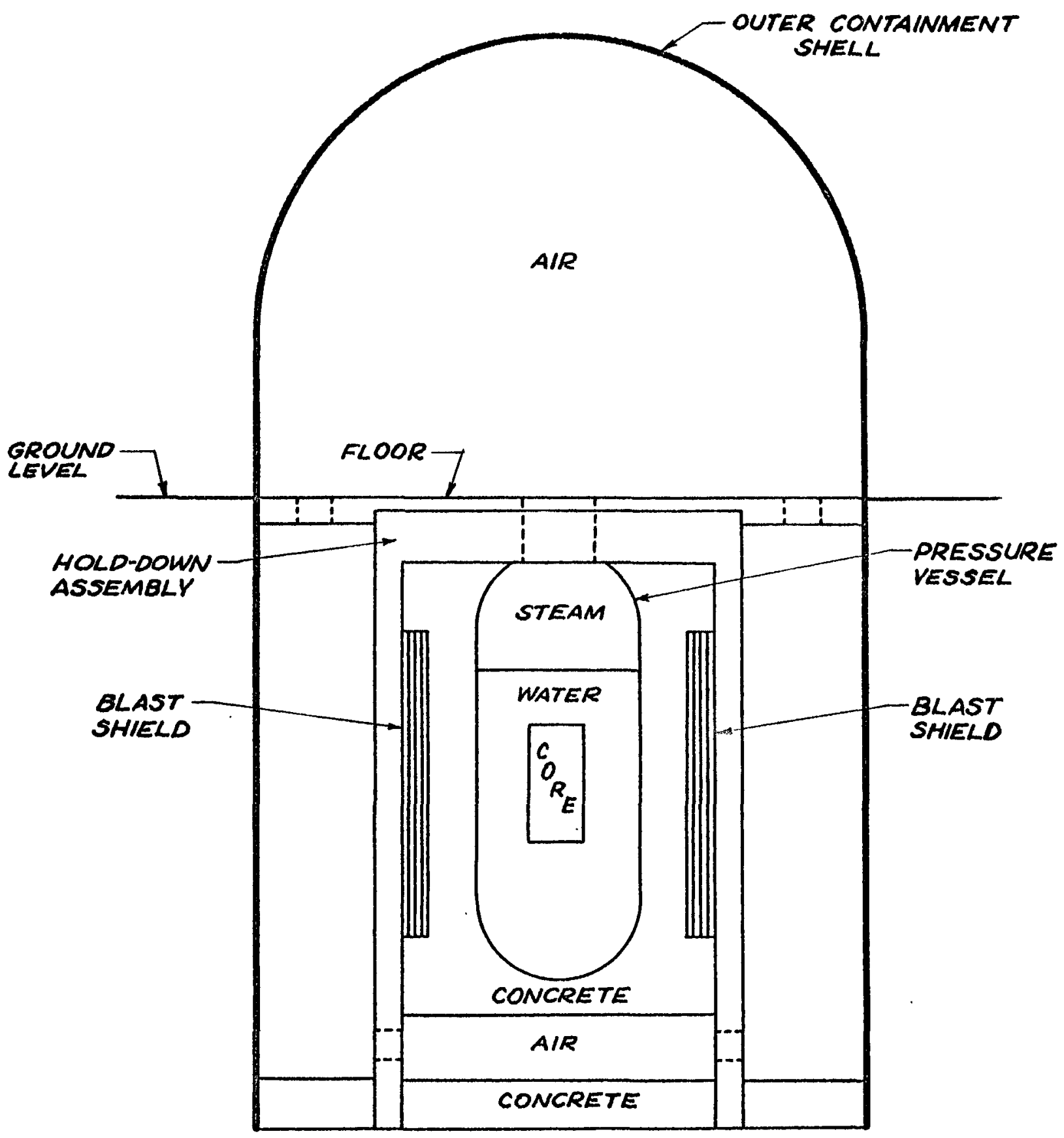

Fig. 7. - IDEALIZED CONPONENTS OF A POWER REACTOR

WTTH REGARD TO CONTAINMENT

-32 . 